Rome, Italy (Mirabella M et al. Neurology April 1992; $\underline{42}$ Suppl $3: 281$ ). There was no muscle weakness and serum CK was only mildly increased.

\title{
HEREDITARY MOTOR AND SENSORY NEUROPATHY MUTATION
}

A duplication in chromosome 17 responsible for most cases of autosomal dominant HMSN 1 was present as a de-novo mutation in 9 out of 10 sporadic patients examined at the Department of Neurology, Academic Medical Center, Amsterdam, The Netherlands. (Hoogendijk JE et al. De-novo mutation in hereditary motor and sensory neuropathy type 1 . Lancet May 2, 1992; 339:1081-82.) (Correspondence: Dr. JE Hoogendijk, Department of Neurology, Academic Medical Center, Meibergdreef 9, 1105 AZ Amsterdam, The Netherlands.)

COMMENT. The previous assumption that isolated cases of HMSN I are most frequently autosomal recessive appears to be incorrect and testing for the duplication in chromosome 17 is essential to establish the mode of inheritance for genetic counseling of isolated patients.

This duplication of part of chromosome 17 was found in affected individuals from 7 of 8 families with HMSN I. Patients with HMSN type II do not show the duplication (Hallam PJ et al. Ann Neurol May 1992; 31:570-572). (Correspondence: Dr. Malcolm, Molecular Genetics Unit, Institute of Child Health, 30 Guilford St., London WC1N 1EH UK.)

HMSN type I also known as Charcot-Marie-Tooth disease type I is known to be genetically heterogeneous. At least 5 genetic loci have been identified including 3 dominant genes and $2 \mathrm{X}$-linked recessive genes. Ionasescu VV et al. from the Department of Pediatrics, University of Iowa Hospitals, Iowa City and the Massachusetts General Hospital present a clinical and genetic linkage study of 8 families with $\mathrm{X}$-linked dominant Charcot-Marie-Tooth neuropathy which supports a localization of the diseased gene between DXS14 and DXYS1 (Neurology April 1992; $\underline{42: 903-908) . ~}$

\section{SEIZURE DISORDERS}

\section{TEMPORAL LOBECTOMY FOR INTRACTABLE SEIZURES}

The results of temporal lobectomy in 16 children under the age of 12 years who had intractable seizures of temporal lobe origin are reported from the Miami Children's Hospital, Florida. All had seizures for at least 2 years despite multiple antiepileptic drugs. Structural lesions were demonstrated by neuroimaging in 11 patients. At follow-up from 1 to 4 years 11 children were seizure free, 3 were $90 \%$ improved, 1 was $50 \%$ improved and 1 was unchanged. Of 9 families contacted 7 reported substantial improvement in the child's psychosocial function. Neuropathological abnormalities identified in all children showed findings consistent with disordered neuronal migration in 7 , tuberous sclerosis in 1 , and mesial temporal sclerosis only in 2 children. (Duchowny $M$ et al. Temporal lobectomy in early childhood. Epilepsia 